\section{TATRA \\ MOUNTaiNS \\ Mathematical Publications}

DOI: $10.2478 /$ tmmp-2013-0022

Tatra Mt. Math. Publ. 55 (2013), 85-94

\title{
REAL FUNCTIONS AND THE EXTENSION OF GENERALIZED PROBABILITY MEASURES
}

\author{
JANA HAVLÍČKOVÁ
}

\begin{abstract}
In the classical probability, as well as in the fuzzy probability theory, random events and probability measures are modelled by functions into the closed unit interval $[0,1]$. Using elementary methods of category theory, we present a classification of the extensions of generalized probability measures (probability measures and integrals with respect to probability measures) from a suitable class of generalized random events to a larger class having some additional (algebraic and/or topological) properties. The classification puts into a perspective the classical and some recent constructions related to the extension of sequentially continuous functions.
\end{abstract}

\section{Introduction}

Sixty years ago, J. N o vák initiated a topological approach to the extension of probability measures from a field of subsets $\mathbb{A}$ of a set $X$ over the generated $\sigma$-field $\sigma(\mathbb{A})$. He pointed out a similarity to the construction of Cech-Stone compactification and developed a theory of sequential envelopes. It is a general theory of the extension of suitable classes of sequentially continuous functions on a sequentially dense subspace over the whole space ([4]-6], [10]-[14], 19]-[27]).

The category $I D$ of $D$-posets of fuzzy sets and sequentially continuous $D$-homomorphisms ([28]) provides a natural background in which various classes of functions into $[0,1]$ are objects and generalized probability measures, observables (dual maps to generalized random variables) are morphisms, and the extension of sequentially continuous maps is intrinsic (categorical), for example, both the extension of measures and the transition from measures to integrals can be viewed as an epireflection ([8], [9], [15]).

(C) 2013 Mathematical Institute, Slovak Academy of Sciences.

2010 Mathematics Subject Classification: 54C20, 28A05, 60A86, 28C99, 06F99.

Keywords: extension of probability measures; outer measure; absolutely measurable set; $D$-poset of fuzzy sets; sequentially continuous $D$-homomorphism; probability integral; $M V$-algebra; Łukasiewicz tribe; classification of extensions; ID-extension; epireflective subcategory. Supported in part by the VEGA grant no. 1/0330/13. 


\section{JANA HAVLÍČKOVÁ}

Using elementary methods of category theory ([1]), we present a classification of the extensions of generalized probability measures (probability measures and integrals with respect to probability measures, states) from a suitable class of generalized random events to a larger class having some additional (algebraic and/or topological) properties. The classification puts into a perspective the classical and some recent constructions related to the extension of sequentially continuous functions and, in particular, measures ([7, [18, [22], [31]).

An interested reader can find more information about generalized probability in [3, 15, [16, 32] and in references therein.

\section{Preliminaries: measure and integral}

Let $\mathbf{A}$ be a field of subsets of a set $X$, let $\mathbf{P}(X)$ be the set of all subsets of $X$, let $\mathcal{P}(\mathbf{A})$ be the set of all probability measures on $\mathbf{A}$, and let $p \in \mathcal{P}(\mathbf{A})$. For each $B \subseteq X$ put

$$
p^{*}(B)=\inf \left\{\sum_{i=1}^{\infty} p\left(A_{i}\right) ; B \subseteq \cup_{i=1}^{\infty} A_{i}, A_{i} \in \mathbf{A}\right\} .
$$

The resulting map $p^{*}: \mathbf{P}(X) \longrightarrow I$ is called induced outer measure. A set $M \subseteq X$ is said to be $p$-measurable whenever for each $B \subseteq X$ we have

$$
p^{*}(B)=p^{*}(B \cap M)+p^{*}\left(B \cap M^{c}\right),
$$

where $M^{c}=X \backslash M$. Denote $\mathbf{M}_{p}$ the set of all $p$-measurable subsets of $X$.

We sum up some facts about $p$-measurable sets (cf. 22], 31]).

Theorem 2.1. Let $\mathbf{A}$ be a field of subsets of a set $X$ and let $p$ be a probability measure on $\mathbf{A}$. Then

(i) $\mathbf{M}_{p}$ is a $\sigma$-field, $\mathbf{A} \subseteq \mathbf{M}_{p}$ and if $B \subseteq X$ and $p^{*}(B)=0$, then $B \in \mathbf{M}_{p}$;

(ii) Define $\bar{p}(B)=p^{*}(B), B \in \mathbf{M}_{p}$. Then $\bar{p}$ is a probability measure on $\mathbf{M}_{p}$ and it is an extension of $p$ over $\mathbf{M}_{p}$;

(iii) $\mathbf{M}_{p}$ is the largest $\sigma$-field of subsets of $X$ which contains $\mathbf{A}$ and on which $p^{*}$ defines a probability measure;

(iv) If $B \subseteq X$, then there exists $A \in \sigma(\mathbf{A}) \subseteq \mathbf{M}_{p}$ such that $B \subseteq A$ and $p^{*}(B)=\bar{p}(A)$.

Denote

$$
\mathbf{M}_{\mathbf{A}}=\bigcap_{p \in \mathcal{P}(\mathbf{A})} \mathbf{M}_{p}
$$

Clearly, $M \subseteq X$ belongs to $\mathbf{M}_{\mathbf{A}}$ if and only if it is $p$-measurable for all $p \in \mathcal{P}(\mathbf{A})$, $\sigma(\mathbf{A}) \subseteq \mathbf{M}_{\mathbf{A}}$, and $\mathbf{M}_{\mathbf{A}}$ is a $\sigma$-field of subsets of the set $X$.

It is known ([21], 23]) that in general we have $\sigma(\mathbf{A}) \neq \mathbf{M}_{\mathbf{A}}$. 
Definition 2.2. Let $\mathbf{A}$ be a field of subsets of a set $X$. Elements of $\mathbf{M}_{\mathbf{A}}$ are said to be absolutely A-measurable sets.

ExAmple 2.3. Let $\mathbf{A}$ be the set of all finite subsets of the closed unit interval $I=[0,1]$ and their complements in $I$. Define a map $p: \mathbf{A} \longrightarrow I$ as follows: if $A \in \mathbf{A}$ is finite, then $p(A)=0$, and otherwise, $p(A)=1$. Then $\mathbf{A}$ is a field of subsets of $I$ and $p$ is a probability measure on $\mathbf{A}$. Denote $\mathbf{I}=\{0,1\}^{I}$ the $\sigma$-field of all subsets of $I$ and $\mathbf{B}$ the $\sigma$-field of all Borel subsets of $I$.

(i) The elements of $\sigma(\mathbf{A})$ are countable subsets of $I$ and their complements in $I$, hence the $\sigma$-field $\mathbf{B}$ is much larger than the $\sigma$-field $\sigma(\mathbf{A})$.

(ii) If $M \subset I$ and $M \notin \sigma(\mathbf{A})$, then both $M$ and $M^{c}$ are uncountable, and

$$
1=p^{*}(I) \neq p^{*}(I \cap M)+p^{*}\left(I \cap M^{c}\right)=1+1 .
$$

Thus $M$ is not $p$-measurable, and hence $\sigma(\mathbf{A})=\mathbf{M}_{p}=\mathbf{M}_{\mathbf{A}}$.

(iii) Denote $q$ the restriction of the Lebesgue measure to $\mathbf{B}$. Then $q$ is an extension of $p$ from $\mathbf{A}$ to $\mathbf{B}$. The sets in $\mathbf{B} \backslash \sigma(\mathbf{A})$ are "out of reach" of the sets in $\mathbf{A}$ and the values $q(A), A \in \mathbf{B} \backslash \sigma(\mathbf{A})$, are "out of reach" of the values $p(A)$, $A \in \mathbf{A}$.

Definition 2.4. Let $\mathbf{A}$ be a field of subsets of a set $X$ and let $p$ be a probability measure on $\mathbf{A}$. Let $\mathbf{B}$ be a field of subsets of a set $X$ such that $\mathbf{A} \subseteq \mathbf{B}$ and let $q$ be a probability measure on $\mathbf{B}$ such that $p(A)=q(A)$ for all $A \in \mathbf{A}$. If

$$
q(B)=\inf \left\{\sum_{i=1}^{\infty} p\left(A_{i}\right) ; B \subseteq \cup_{i=1}^{\infty} A_{i}, A_{i} \in \mathbf{A}\right\}
$$

for all $B \in \mathbf{B}$, then $q$ is said to be a measurable extension of $p$.

Let $q$ be a measurable extension of $p \in \mathcal{P}(\mathbf{A})$ on $\mathbf{A}$ over $\mathbf{M}_{\mathbf{A}}$. Clearly, for all $M \in \mathbf{M}_{\mathbf{A}}$ we have $q(M)=p^{*}(M)$.

\section{D-posets of fuzzy sets}

$D$-posets have been introduced in [17] in order to model events in quantum probability. They generalize Boolean algebras, $M V$-algebras and other probability domains, and provide a category in which observables and states become morphisms $([2,8])$. Recall that a $D$-poset is a partially ordered set with the greatest element $1_{X}$, the least element $0_{X}$, and a partial binary operation called difference, such that $a \ominus b$ is defined if and only if $b \leq a$, and the following axioms are assumed:

(D1) $a \ominus 0_{X}=a$ for each $a \in X$;

(D2) If $c \leq b \leq a$, then $a \ominus b \leq a \ominus c$ and $(a \ominus c) \ominus(a \ominus b)=b \ominus c$. 


\section{JANA HAVLÍČKOVÁ}

A canonical example is the interval $[0,1]=I$ (linearly ordered, $a \ominus b=a-b$ whenever $b \leq a)$. Fundamental to applications $([15], 28])$ are $D$-posets of fuzzy sets, i.e., systems $\mathcal{X} \subseteq I^{X}$ carrying the coordinatewise partial order, coordinatewise convergence of sequences, containing the top and bottom elements of $I^{X}$, and closed with respect to the partial operation difference defined coordinatewise. We always assume that $\mathcal{X}$ is reduced, i.e., for $x, y \in X, x \neq y$, there exists $u \in \mathcal{X}$ such that $u(x) \neq u(y)$. Denote $I D$ the category having (reduced) $D$-posets of fuzzy sets as objects and having sequentially continuous $D$-homomorphisms (preserving constants, order, and the difference) as morphisms. Objects of $I D$ are subobjects of the powers $I^{X}$.

Recall that each $D$-poset can be reorganized into an effect algebra and the two structures are equivalent (cf. [3], 29]).

Concerning the undefined notions, the reader is referred to [1] and [3].

$\operatorname{Let}(X, \mathbf{A}, p)$ be a classical probability space. Denote $\mathcal{M}(\mathbf{A})$ the system of all measurable functions into $[0,1]=I$. Then $\mathbf{A}$ (as indicator functions) and $\mathcal{M}(\mathbf{A})$ are $D$-posets of fuzzy sets, $p$ and the probability integral $\tilde{p}(f)=\int f d p$ are sequentially continuous $D$-homomorphisms (remember the Lebesgue Dominated Convergence Theorem). What is more important and surprising, the following assertions hold (cf. [8]).

Theorem 3.1.

(i) Let $p$ be a sequentially continuous D-homomorphism of $\mathbf{A}$ into I. Then $p$ is a probability measure.

(ii) Let $h$ be a sequentially continuous D-homomorphisms of $\mathcal{M}(\mathbf{A})$ into $I$. Then $h$ is a probability integral, i.e., there exists a probability measure $p$ on $\mathbf{A}$ such that $h(f)=\int f d p, f \in \mathcal{M}(\mathbf{A})$.

(iii) Let $(Y, \mathbf{B})$ be a measurable space. Then each sequentially continuous D-homomorphism on $\mathbf{B}$ into $\mathcal{M}(\mathbf{A})$ can be uniquely extended to a sequentially continuous D-homomorphism on $\mathcal{M}(\mathbf{B})$ into $\mathcal{M}(\mathbf{A})$.

Observe that if $\{a\}$ is a singleton and $\mathbf{T}=\{\emptyset,\{a\}\}$ is the corresponding trivial field of all subsets of $\{a\}$, then $I$ and $\mathcal{M}(\mathbf{T})$ coincide.

\section{Classification of extensions}

Let $\mathcal{X}, \mathcal{Y} \subseteq I^{X}$ be D-posets of fuzzy sets, i.e., objects of $I D$. If $\mathcal{X} \subseteq \mathcal{Y}$, then $\mathcal{Y}$ is said to be an extension of $\mathcal{X}$. For example, let $\mathbf{A} \subseteq\{0,1\}^{X}$ be a field of sets, then $\sigma(\mathbf{A}), \mathbf{M}_{\mathbf{A}}$, and $\mathcal{M}(\sigma(\mathbf{A}))$ are canonical extensions of $\mathbf{A}$.

In this section, we study extensions of $D$-posets of fuzzy sets. Motivated by the canonical extensions, we introduce a classification of extensions in terms 
of subcategories of $I D$ to which the extension belongs and in terms of some additional properties of the extension.

For $u, v \in I^{X}$, define operations $\wedge$ and $\vee$ coordinatewise: $(u \wedge v)(x)=u(x) \wedge$ $v(x)$ and $(u \vee v)(x)=u(x) \vee v(x), x \in X$. D-posets of fuzzy sets closed with respect to $\wedge$ and $\vee$ play an important role in generalized probability. Indeed, if a $D$-poset of fuzzy sets $\mathcal{X} \subseteq I^{X}$ is closed with respect to $\wedge$ and $\vee$, then (cf. [15]) $\mathcal{X}$ is a bold algebra, i.e., an $M V$-algebra representable by fuzzy sets. Recall that $M V$-algebras generalize Boolean algebras and play an important role in manyvalued logic and generalized probability (cf. 30]). A bold algebra $\mathcal{X} \subseteq I^{X}$ which is sequentially closed in $I^{X}$ is said to be a Eukasiewicz tribe. Observe that fields of sets are bold algebras and $\sigma$-fields and measurable $I$-valued functions are Eukasiewicz tribes. Further, if $\mathcal{X} \subseteq I^{X}$ is a Eukasiewicz tribe, then there exists a $\sigma$-field $\mathbf{A} \subseteq\{0,1\}^{X}$ such that $\mathbf{A} \subseteq \mathcal{X} \subseteq \mathcal{M}(\mathbf{A})$ and $\mathcal{X}=\mathcal{M}(\mathbf{A})$ whenever $\mathcal{X}$ contains all constant $I$-valued functions (cf. [31]).

Let $\mathcal{X} \subseteq I^{X}$ be a D-poset of fuzzy sets. Denote $\mathcal{S}(\mathcal{X})$ the set of all sequentially continuous $D$-homomorphisms of $\mathcal{X}$ into $I$; the elements of $\mathcal{S}(\mathcal{X})$ are called states. Let $\mathbf{A} \subseteq\{0,1\}^{X}$ be a field of subsets of $X$. Denote $\mathcal{P}(\mathbf{A})$ the set of all probability measures on $\mathbf{A}$. It is known (cf. [8]) that $\mathcal{S}(\mathbf{A})=\mathcal{P}(\mathbf{A})$.

In our classification we will consider several (full) subcategories of $I D$ : the objects of FSD are fields of sets, the objects of $C F S D$ are $\sigma$-fields of sets, the objects of $A C F S D$ are $\sigma$-fields of sets of the form $\mathbf{M}_{\mathbf{A}}$ (absolutely measurable sets), the objects of $B I D$ are bold algebras, the objects of $C B I D$ are Eukasiewicz tribes, the objects of $C G B I D$ are $D$-posets od fuzzy sets of the form $\mathcal{M}(\mathbf{A})$ where $\mathbf{A}$ is a $\sigma$-field of subsets.

Definition 4.1. Let $\mathcal{X}, \mathcal{Y} \subseteq I^{X}$ be D-posets of fuzzy sets, $\mathcal{X} \subseteq \mathcal{Y}$. Let $\mathcal{H}(\mathcal{X})$ be a subset of $\mathcal{S}(\mathcal{X})$. If for each $s \in \mathcal{H}(\mathcal{X})$ there exists $t \in \mathcal{S}(\mathcal{Y})$ such that $s(u)=t(u)$ for all $u \in \mathcal{X}$, then $\mathcal{X}$ is said to be $\mathcal{H}(\mathcal{X})$-embedded in $\mathcal{Y}$.

ExAmple 4.2. Let $\mathbf{A} \subseteq\{0,1\}^{X}$ be a field of sets and let $\sigma(\mathbf{A})$ be the generated $\sigma$-field. Then $\sigma(\mathbf{A})$ as an extension has several interesting properties (cf. 6, 7 , [8], 27]).

(i) $\mathbf{A}=\sigma(\mathbf{A})$ if and only if $\mathbf{A}$ is sequentially closed in $I^{X}$ (recall that $A=\lim _{n \rightarrow \infty} A_{n}$ in $I^{X}$ if $A=\limsup A_{n}=\liminf A_{n}$, where $\limsup A_{n}=$ $\bigcap_{k=1}^{\infty} \bigcup_{n=k}^{\infty} A_{n}$ and $\lim \inf A_{n}=\bigcup_{k=1}^{\infty} \bigcap_{n=k}^{\infty} A_{n}$ or, equivalently, the sequence $\left\{\chi_{A_{n}}\right\}_{n=1}^{\infty}$ of indicator functions converges coordinatewise to the indicator function $\left.\chi_{A}\right)$, i.e., if and only if $\mathbf{A}$ is a Eukasiewicz tribe.

(ii) $\mathbf{A}$ is $\mathcal{P}(\mathbf{A})$-embedded in $\sigma(\mathbf{A})$.

(iii) A is sequentially dense in $\sigma(\mathbf{A})$ and $\sigma(\mathbf{A})$ is the smallest of all sequentially closed fields of subsets of $X$ containing $\mathbf{A}$. Indeed, for a system $\mathbf{B}$ 


\section{JANA HAVLÍČKOVÁ}

of subsets of $X$ define $\operatorname{cl}(\mathbf{B}) \subseteq \mathbf{P}(X)$ as the set of all sequential limits of sequences of sets in $\mathbf{B}$ and, inductively, for each ordinal number $\alpha \leq \omega_{1}$ define

$$
\mathrm{cl}^{0}(\mathbf{B})=\mathbf{B}, \quad \operatorname{cl}^{\alpha}(\mathbf{B})=\operatorname{cl}\left(\mathrm{cl}^{\alpha-1}(\mathbf{B})\right)
$$

for an isolated ordinal number,

$$
\operatorname{cl}^{\alpha}(\mathbf{B})=\operatorname{cl}\left(\bigcup_{\beta<\alpha} \operatorname{cl}^{\beta}(\mathbf{B})\right)
$$

for a limit ordinal number; it is known (cf. [27]) that each $\mathrm{cl}^{\alpha}$ is a closure operator (need not be idempotent), $\mathrm{cl}^{\omega_{1}}$ is a topological closure operator (topology), each $\mathrm{cl}^{\alpha}(\mathbf{A})$ is a field of subsets of $X$, and $\mathrm{cl}^{\omega_{1}}(\mathbf{A})=\sigma(\mathbf{A})$. If $\mathrm{cl}^{\alpha}(\mathbf{B})=\mathbf{C}$ for some ordinal number $\alpha$, then $\mathbf{B}$ is said to be sequentially dense in $\mathbf{C}$.

(iv) $\sigma(\mathbf{A})$ is the largest of all fields of subsets of $X$ in which $\mathbf{A}$ is $\mathcal{P}(\mathbf{A})$ -embedded and sequentially dense. Observe that $\mathbf{A}$ is $\mathcal{P}(\mathbf{A})$-embedded (see (ii)) and sequentially dense (see (iii)) in $\sigma(\mathbf{A})$. Since $\sigma(\mathbf{A})$ is sequentially closed (see (i)), there is no larger field of subsets of $X$ in which $\mathbf{A}$ is sequentially dense.

(v) Each probability measure $p \in \mathcal{P}(\mathbf{A})$ can be uniquely extended to a probability measure $\bar{p} \in \mathcal{P}(\sigma(\mathbf{A}))$. Indeed (see (ii)), let $\bar{p} \in \mathcal{P}(\sigma(\mathbf{A}))$ be an extension of $p$. If $q \in \mathcal{P}(\sigma(\mathbf{A}))$ is an extension of $p$, then $\{A \in \sigma(\mathbf{A}) ; \bar{p}(A)=q(A)\}$ contains $\mathbf{A}$ and it is sequentially closed in $I^{X}$ (two sequentially continuous functions coincide on a sequentially closed domain-possibly empty, see [26]). Finally, $\sigma(\mathbf{A})$ is the smallest sequentially closed system of subsets of $X$ containing $\mathbf{A}$ (see (ii)).

(vi) Let $h$ be a sequentially continuous $D$-homomorphism of $\mathbf{A}$ into a $\sigma$-field $\mathbf{B} \subseteq\{0,1\}^{Y}$. Then $h$ can be uniquely extended to a sequentially continuous $D$-homomorphism $h_{\sigma}$ of $\sigma(\mathbf{A})$ into B. Consequently, $C F S D$ is an epireflective subcategory of FSD (cf. [6]).

Example 4.3. Let $\mathbf{A} \subseteq\{0,1\}^{X}$ be a field of sets and let $\mathbf{M}_{\mathbf{A}}$ be the $\sigma$-field of absolutely A-measurable subsets of $X$. Then

(i) $\mathbf{M}_{\mathbf{A}}$ is the largest $\sigma$-field of subsets of $X$ in which $\mathbf{A}$ is $\mathcal{P}(\mathbf{A})$-embedded (see (iii) in Theorem 2.1);

(ii) Each probability measure $p \in \mathcal{P}(\mathbf{A})$ has a unique measurable extension $\bar{p} \in \mathcal{P}\left(\mathbf{M}_{\mathbf{A}}\right)$ (see the end of Section 2).

Definition 4.4. Let $\mathcal{X} \subseteq I^{X}$ be a D-poset of fuzzy sets and let $n$ be a natural number, $n>1$. Assume that for each $u \in \mathcal{X}, u \neq 0$, there exists an element $u(n) \in \mathcal{X}$ such that $0<u(n)<u$ and, for each $k=1,2, \ldots, n-1$, we can subtract from $u$ successively $k$-times $u(n)$ and the result is greater or equal to $u(n)$, and if we subtract from $u$ successively $n$-times $u(n)$, then the result is 0 . Then $\mathcal{X}$ is said to be divisible by $n$. If $\mathcal{X}$ is divisible by $n$ for each natural number $n, n>1$, then $\mathcal{X}$ is said to be divisible. 
ExAmple 4.5. Let $\mathbf{A} \subseteq\{0,1\}^{X}$ be a field of sets and let $\sigma(\mathbf{A})$ be the generated $\sigma$-field. Consider the $D$-poset $\mathcal{M}(\sigma(\mathbf{A}))$ of all measurable functions ranging in $[0,1]$ as an extension of $\mathbf{A}$. Then

(i) $\mathcal{M}(\sigma(\mathbf{A}))$ is a divisible extension of $\mathbf{A}$;

(ii) $\mathcal{M}(\sigma(\mathbf{A}))$ is the smallest of all divisible extensions of $\mathbf{A}$ which are bold algebras sequentially closed in $I^{X}$. Indeed, let $\mathcal{X} \subseteq I^{X}$ be a divisible extension of $\mathbf{A}$ and assume that $\mathcal{X}$ is a bold algebra sequentially closed in $I^{X}$. Then $\mathcal{X}$ is a Eukasiewicz tribe and contains all constant functions ranging in $I$. It is known (cf. [15], [31]), that if $\mathcal{X} \subseteq I^{X}$ is a Eukasiewicz tribe, then there exists a unique $\sigma$-field $\mathbf{B} \subseteq\{0,1\}^{X}$ such that $\mathbf{B} \subseteq \mathcal{X} \subseteq \mathcal{M}(\mathbf{B})$ and if $\mathcal{X}$ contains all constant functions ranging in $I$, then $\mathcal{X}=\overline{\mathcal{M}}(\mathbf{B})$. From $\mathbf{A} \subseteq \mathcal{X}=\mathcal{M}(\mathbf{B})$ it follows that

$$
\mathbf{A} \subseteq \sigma(\mathbf{A}) \subseteq \mathbf{B} \subseteq \mathcal{X} \quad \text { and } \quad \mathcal{M}(\sigma(\mathbf{A})) \subseteq \mathcal{X}=\mathcal{M}(\mathbf{B})
$$

(iii) $\mathbf{A}$ is $\mathcal{P}(\mathbf{A})$-embedded in $\mathcal{M}(\sigma(\mathbf{A}))$. The assertion follows from the fact that if $p \in \mathcal{P}(\mathbf{A})$, then the probability integral with respect to $p$ is a sequentially continuous $D$-homomorphism on $\mathcal{M}(\sigma(\mathbf{A}))$ into $I$;

(iv) Each probability measure $p \in \mathcal{P}(\mathbf{A})$ can be uniquely extended to a sequentially continuous $D$-homomorphism of $\mathcal{M}(\sigma(\mathbf{A}))$ into $I$. The assertion follows from the fact that each sequentially continuous $D$-homomorphism on $\mathcal{M}(\sigma(\mathbf{A}))$ into $I$ coincides with a unique probability integral (see (ii) in Theorem 3.1);

(v) Let $\mathbf{B}$ be a $\sigma$-field of subsets of a set $Y$, let $\mathcal{M}(\mathbf{B})$ be the $D$-poset of all measurable functions ranging in $I$, and let $h$ be a sequentially continuous $D$-homomorphism of $\sigma(\mathbf{A})$ into $\mathcal{M}(\mathbf{B})$. Then $h$ can be uniquely extended to a sequentially continuous $D$-homomorphism $\bar{h}$ of $\mathcal{M}(\sigma(\mathbf{A}))$ into $\mathcal{M}(\mathbf{B})$ (cf. 8 , Theorem 4.2]);

(vi) $C B I D$ is an epireflective subcategory of $C G B I D$ (cf. [15, Corollary 4.4]).

Motivated by the canonical extensions, we divide the properties of extensions (boldface) of $D$-posets of fuzzy sets into two groups - internal and external. Let $\mathcal{X}, \mathcal{Y}$ be $D$-posets of fuzzy sets such that $\mathcal{X} \subseteq \mathcal{Y} \subseteq I^{X}$, i.e., let $\mathcal{Y}$ be an extension of $\mathcal{X}$.

Internal. The following properties of $\mathcal{Y}$ are in terms of the structure of $I^{X}$ :

(i) $\mathcal{Y}$ is closed with respect to $\wedge$ and $\vee$;

(ii) $\mathcal{Y}$ is divisible;

(iii) $\mathcal{X}$ is sequentially dense in $\mathcal{Y}$;

(iv) $\mathcal{Y}$ is sequentially closed in $I^{X}$.

External. The following properties of $\mathcal{Y}$ are in terms of extension of morphisms belonging to a suitable class:

(i) $\mathcal{X}$ is $\mathcal{H}(\mathcal{X})$-embedded in $\mathcal{Y}$;

(ii) the extensions of morphisms in question are uniquely determined; 


\section{JANA HAVLÍČKOVÁ}

(iii) $\mathcal{Y}$ is a largest or smallest of all extensions of $\mathcal{X}$ to which the morphisms in question can be extended;

(iv) $I D_{1}$ and $I D_{2}$ are subcategories of $I D, I D_{2}$ is epireflective in $I D_{1}, \mathcal{X}$ is an object of $I D_{1}, \mathcal{Y}$ is an object of $I D_{2}$, and $\mathcal{Y}$ is the epireflection of $\mathcal{X}$.

Definition 4.6. Let $\mathcal{X}, \mathcal{Y} \subseteq I^{X}$ be D-posets of fuzzy sets, i.e., objects of $I D$. If $\mathcal{X} \subseteq \mathcal{Y}$, then $\mathcal{Y}$ is said to be an $I D$-extension of $\mathcal{X}$.

$I D$-extensions and the internal and external properties provide a natural language for the construction and classification of generalized probability domains. In particular, each canonical extension can be characterized as an $I D$-extension having suitable properties. Such approach to probability theory leads to a better understanding of classical probability theory, fuzzy probability theory, and their mutual relationship (cf. [15]).

We close with two problems and a suggestion related to future research in the area of extensions of $D$-posets of fuzzy sets.

Problem 1. Is $A C F S D$ an epireflective subcategory of $F S D$ ?

Problem 2. Is $C G B I D$ an epireflective subcategory of $B I D$ ?

Definition 4.7. Let $I D_{0}$ be a subcategory of $I D$ and let $\mathcal{A} \subseteq I^{A}$ be an object of $I D_{0}$. Let $\mathcal{X}, \mathcal{Y}$ be objects of $I D_{0}$ such that $\mathcal{X} \subseteq \mathcal{Y} \subseteq I^{X}$. If each morphism $f$ of $\mathcal{X}$ into $\mathcal{A}$ can be uniquely extended to a morphism $f_{\mathcal{Y}}$ of $\mathcal{Y}$ into $\mathcal{A}$, then $\mathcal{Y}$ is said to be an $\mathcal{A}$-extension of $\mathcal{X}$. If $\mathcal{X}$ has no proper $\mathcal{A}$-extension, then $\mathcal{X}$ is said to be $\mathcal{A}$-absolute. If $\mathcal{Y}$ is an $\mathcal{A}$-extension of $\mathcal{X}$ and $\mathcal{Y}$ is $\mathcal{A}$-absolute, then $\mathcal{Y}$ is said to be an $\mathcal{A}$-envelope of $\mathcal{X}$.

It might be interesting to develop a theory of $\mathcal{A}$-envelopes for suitable subcategories $I D_{0}$ of $I D$.

\section{REFERENCES}

[1] ADÁMEK, J.: Theory of Mathematical Structures. Reidel, Dordrecht, 1983.

[2] CHOVANEC, F.-KÔPKA, F.: D-posets, in: Handbook of Quantum Logic and Quantum Structures: Quantum Structures (K. Engesser et al., eds.), Elsevier, Amsterdam, 2007, pp. $367-428$.

[3] DVUREČEnSKiJ, A.-PUlmannovÁ, S.: New Trends in Quantum Structures, in: Math. Appl. (Dordrecht), Vol. 516, Kluwer Acad. Publ. Dordrecht; Ister Science, Bratislava, 2000.

[4] FRIČ, R.: Remarks on sequential envelopes, Rend. Istit. Math. Univ. Trieste 20 (1988), $19-28$.

[5] FRIČ, R.: History of sequential convergence spaces, in: Handbook of the History of General Topology, Vol. 1 (C. E. Aull et al., eds.), Kluwer Acad. Publ., Amsterdam, 1997, pp. 343-355. 


\section{REAL FUNCTIONS AND THE EXTENSION OF GENERALIZED PROBABILITY MEASURES}

[6] FRIČ, R.: Eukasiewicz tribes are absolutely sequentially closed bold algebras, Czechoslovak Math. J. 52 (2002), 861-874.

[7] FRIČ, R.: Extension of measures: a categorical approach, Math. Bohemica 130 (2005), 397-407.

[8] FRIČ, R.: Extension of domains of states, Soft Comput. 13 (2009), 63-70.

[9] FRIČ, R.: Measures: continuity, measurability, duality, extension, Tatra Mt. Math. Publ. 42 (2009), 161-174.

[10] FRIČ, R.: From probability to sequences and back Rend. Istit. Mat. Univ. Trieste 44 (2012), 285-296.

[11] FRIČ, R.-HUŠEK, M.: Projectively generated convergence of sequences, Czechoslovak Math. J. 33 (1983), 525-536.

[12] FRIČ, R.-KEMOTO, N.: Sequential envelope revisited, Abstracts of the 8th Prague Topological Symposium, Part B: Extended abstracts, Prague, 1996, pp. 126-134.

[13] FRIČ, R.-KENT, D. C.: The finite product theorem for certain epireflections, Math. Nachr. 150 (1991), 7-14.

[14] FRIČ, R.-MCKENNON, K.-RICHARDSON, G. D.: Sequential convergence in $C(X)$, in: Convergence Structures and Applications to Analysis, Frankfurt/Oder, 1978, Abh. Akad. Wiss. DDR, Abt. Math. Natur. Technik, Vol. 1979, Akademie-Verlag, Berlin, 1980, pp. $56-65$.

[15] FRIČ, R.-PAPČO, M.: On probability domains II, Internat. J. Theoret. Phys. 50 (2011), 3778-3786.

[16] GUDDER, S.: Fuzzy probability theory, Demonstratio Math. 31 (1998), 235-254.

[17] KÔPKA, F.-CHOVANEC, F.: D-posets, Math. Slovaca 44 (1994), 21-34.

[18] JUREČKOVÁ, M.: The measure extension theorem for MV-algebras, Tatra Mt. Math. Publ. 6 (1995), 56-61.

[19] KENT, D. C.-RICHARDSON, G. D.: Two generalizations of Novák's sequential Envelope, Math. Nachr. 19 (1979), 77-85.

[20] KRATOCHVÍL, P.: Multisequences and measure, in: General Topology and its Relations to Modern Analysis and Algeba IV, Proc. 4th Prague Topological Sympos., Prague, 1976, Society of Czechoslovak Math. and Phys., Prague, pp. 237-244.

[21] LAVRENTIEV, M.: Contributions a la théorie des ensembles homéomorphes, Fund. Math. 6 (1924), 149-160.

[22] LOYA, P.: Measure and probability theory: countable additivity, (http://www.math.binghamton.edu/paul/505-S08/505-7.pdf)

[23] MARCZEWSKI (-SZPILRAJN), E.: On absolutely measurable sets and functions, in: Collected Mathematical Papers, Polish Academy of Sciences, Warszaw, 1966, pp. 160-18.

[24] NOVÁK, J.: Über die eindeutigen stetigen Erweiterungen stetiger Funktionen, Czechoslovak Math. J. 8 (1958), 344-355.

[25] NOVÁK, J.: On the sequential envelope, in: General Topology and its Relations to Modern Analysis and Algeba, Proc. 1st Prague Topological Sympos., Prague, 1961, Publishing House of the Czechoslovak Academy of Sciences, Prague, 1962, pp. 292-294.

[26] NOVÁK, J.: On convergence spaces and their sequential envelopes, Czechoslovak Math. J. 15 (1965), 74-100.

[27] NOVÁK, J.: On sequential envelopes defined by means of certain classes of functions, Czechoslovak Math. J. 18 (1968), 450-456.

[28] PAPČO, M.: On measurable spaces and measurable maps, Tatra Mt. Math. Publ. 28 (2004), 125-140. 


\section{JANA HAVLÍČKOVÁ}

[29] PAPČO, M.: On effect algebras, Soft Comput. 12 (2007), 26-35.

[30] RIEČAN, B.-MUNDICI, D.: Probability on $M V$-algebras, in: Handbook of Measure Theory, Vol. II (E. Pap, ed.), North-Holland, Amsterdam, 2002, pp. 869-910.

[31] RIEČAN, B.-NEUBRUNN, T.: Integral, Measure, and Ordering. Kluwer Acad. Publ., Dordrecht, 1997.

[32] ZADEH, L. A.: Probability measures of fuzzy events, J. Math. Anal. Appl. 23 (1968), $421-427$.

Received May 5, 2013

Faculty of Mathematics

Physics and Informatics

Comenius University

Mlynská dolina

SK-842-48 Bratislava

SLOVAKIA

E-mail: jani.havlickova@gmail.com 